\title{
Association between aspirin use and lung cancer incidence depends on high-frequency use, bodyweight, and age in U.S. adults
}

\author{
Xuesi Dong ${ }^{1,2,3,4 \#}$, Jieyu He ${ }^{1,3 \#}$, Lijuan Lin ${ }^{1,2,3 \#}$, Ying Zhu ${ }^{1,3}$, Chao Chen ${ }^{1,3}$, Li Su, ${ }^{2,5}$, Yang Zhao ${ }^{1,2,3,6}$, \\ Ruyang Zhang ${ }^{1,2,3,6}$, Yongyue Wei ${ }^{1,2,3,7}$, Feng Chen ${ }^{1,3,8,9 *}$, David C. Christiani ${ }^{2,5 *}$
}

${ }^{1}$ Department of Biostatistics, Center for Global Health, School of Public Health, Nanjing Medical University, Nanjing, China; ${ }^{2}$ Department of Environmental Health, Harvard T.H. Chan School of Public Health, Boston, MA, USA; ${ }^{3}$ Key Laboratory of Biomedical Big Data of Nanjing Medical University, Nanjing, China; ${ }^{4}$ Department of Epidemiology and Biostatistics, School of Public Health, Southeast University, Nanjing, China; ${ }^{5}$ Pulmonary and Critical Care Division, Department of Medicine, Massachusetts General Hospital and Harvard Medical School, Boston, MA, USA; ${ }^{6}$ Department of Medical Oncology, Jinling Hospital, School of Medicine, Nanjing University, Nanjing, China; ${ }^{7}$ China International Cooperation Center for Environment and Human Health, Nanjing Medical University, Nanjing, China; ${ }^{8}$ State Key Laboratory of Reproductive Medicine, Nanjing Medical University, Nanjing, China; 'Jiangsu Key Lab of Cancer Biomarkers, Prevention and Treatment, Cancer Center, Collaborative Innovation Center for Cancer Personalized Medicine, Nanjing Medical University, Nanjing, China

Contributions: (I) Conception and design: X Dong, R Zhang, Y Wei; (II) Administrative support: F Chen, DC Christiani; (III) Provision of study materials or patients: Y Zhu; (IV) Collection and assembly of data: X Dong, Y Zhu; (V) Data analysis and interpretation: X Dong, J He, L Lin; (VI) Manuscript writing: All authors; (VII) Final approval of manuscript: All authors.

"These authors contributed equally to this work.

*These authors contributed equally to this work as senior authors.

Correspondence to: Ruyang Zhang, PhD. SPH Building Room 406, 101 Longmian Avenue, Nanjing 211166, China; Email: zhangruyang@njmu.edu.cn; Yongyue Wei, PhD. SPH Building Room 418, 101 Longmian Avenue, Nanjing 211166, China. Email: ywei@njmu.edu.cn; Feng Chen, PhD. SPH Building Room 410, 101 Longmian Avenue, Nanjing 211166, China. Email: fengchen@njmu.edu.cn.

Backgroundk Chemoprevention of cancer with aspirin is controversial as a primary prevention strategy. We sought to investigate the association between aspirin frequency and risk of lung cancer in The Prostate, Lung, Colorectal and Ovarian (PLCO) Cancer Screening Trial.

Methods: Using data from 101,722 participants in PLCO, we used a Cox regression model coupling with propensity score to detect the association between aspirin frequency and lung cancer risk.

Results: High-frequency aspirin use significantly increased risk of lung cancer by $28 \%$ compared to no use $\left(\mathrm{HR}=1.28 ; 95 \% \mathrm{CI}, 1.14-1.45 ; \mathrm{P}=3.37 \times 10^{-5}\right)$, especially for current smoker $(\mathrm{HR}=1.30 ; 95 \%$ $\left.\mathrm{CI}, 1.07-1.57 ; \mathrm{P}=6.82 \times 10^{-3}\right)$. However, the increased lung cancer risk due to high-frequency aspirin use significantly decreased with increasing bodyweight $\left(\mathrm{HR}_{\text {interaction }}=0.96 ; 95 \% \mathrm{CI}, 0.94-0.99 ; \mathrm{P}=1.26 \times 10^{-2}\right)$. Further, for participants with bodyweight $<80 \mathrm{~kg}$, high-frequency aspirin use showed an elevated risk at $<76$ years of age $\left(\mathrm{HR}_{\text {age }<76}=1.47 ; 95 \% \mathrm{CI}, 1.25-1.73 ; \mathrm{P}=3.81 \times 10^{-6}\right)$. Our study used propensity score under various confounding and stratification analyses by cardio-cerebrovascular status, which all presented similar evidences.

Conclusions: High-frequency aspirin use is associated with the increased risk of lung cancer. Current smoker or people with age $<76$ years and bodyweight $<80 \mathrm{~kg}$ should be more cautious to highfrequency aspirin use for lung cancer chemoprevention. This study provides a new insight for lung cancer chemoprevention.

Keywords: Aspirin; lung cancer; risk; bodyweight; age

Submitted Mar 10, 2020. Accepted for publication Sep 19, 2020.

doi: $10.21037 /$ tlcr-20-414

View this article at: http://dx.doi.org/10.21037/tlcr-20-414 


\section{Introduction}

Aspirin, one of the most frequently used medications worldwide, is effective for treatment of pain, inflammation, and fever (1). Recently, chemoprevention of cancer with aspirin has become of particular interest as a primary prevention strategy (2). This function may be due to the role of aspirin in inhibiting both non-selectively inactivating cyclooxygenase (COX)-1 and COX-2 enzymes, resulting in restoration of normal apoptosis and reduction of angiogenesis (3).

However, the practice of using aspirin for primary prevention of cancer remains controversial. A recent largescale cohort study shown that long-term aspirin use was associated with a modest reduced risk for overall cancer, especially gastrointestinal tract tumors; regular aspirin use may prevent a substantial proportion of colorectal cancers and complement the benefits of screening (4). Consistently, United States Preventive Services Task Force recommends low-dose aspirin use for colorectal cancer chemoprevention in adults ages 50 to 59 years and 60 to 69 years, with grade level $\mathrm{B}$ and $\mathrm{C}$ respectively (5). However, a recent study demonstrated that risk of death from any cause is higher in aspirin users, with cancer a major contributor to higher mortality in the aspirin-using group (6). The American Cancer Society recommends against aspirin use for colorectal cancer prevention in the general population (7), except for persons with Lynch syndrome (8). Additionally, another study showed a slight promotion of brain tumors with aspirin use (9). These negative reports raise public concern over aspirin chemoprophylaxis.

For lung cancer, several meta-analyses demonstrate varied results and interpretations. Some studies show that aspirin users have significantly lower risk, while others report no significant difference (10-14). With adequate adjustment for demographic, dietary, and ethological factors, analysis of a cohort of women in the U.S. showed a $55 \%$ increased risk of lung cancer with high-frequency aspirin use $(\geq 15$ tablets per week) (15). On the other hand, evidences shown that the effect of aspirin use on chemoprevention can be modified by bodyweight and age $(16,17)$. Thus, adequate adjustment for covariates (e.g., demographic, diet, cardiocerebrovascular disease history) in a statistical model and comprehensive consideration of modifiers (e.g., bodyweight, age) may account for varied reported results. Further, the modified effect of high-frequency aspirin use on lung cancer risk by both bodyweight and age remains largely unclear.

Therefore, we analyzed data from participants enrolled in the Prostate, Lung, Colorectal and Ovarian (PLCO) study to evaluate the association between aspirin use and lung cancer with the modifier roles of bodyweight and age as well as adjustment for demographic, dietary, and ethological factors. The results of our study provide new insight into the use of aspirin for chemoprevention of lung cancer.

We present the following article in accordance with the STROBE reporting checklist (available at http://dx.doi. org/10.21037/tlcr-20-414).

\section{Methods}

\section{Experimental design}

The study design of PLCO has been described previously (18). Briefly, 154,897 participants aged 55-74 years were enrolled during November 1993-July 2001, and data were collected up to December 31, 2009, with median follow-up of 11.9 years. The study was conducted in accordance with the Declaration of Helsinki (as revised in 2013). All participants provided written informed consent, and the study was approved by both the National Cancer Institute and local Institutional Review Boards (ClinicalTrials.gov registration numbers: NCT01696968). We extracted Diet History Questionnaire (DHQ) data from PLCO, which was offered to both arms of the trial (screening and control) starting in 1998. In total, $77 \%$ of all participants in both arms of the trial completed the DHQ, screening arm complete $78 \%$, and control $\operatorname{arm} 75 \%$, respectively. Data for demographic and dietary variables were collected through epidemiological questions in a baseline questionnaire (BQ). We sequentially excluded unqualified samples for the following reasons: missing BQ; incomplete DHQ; invalid DHQ [missing $\geq 8$ food items or extreme energy intake values (i.e., lowest or highest $1 \%$ )]; cancer diagnosed before study entry. After these exclusions, 101,722 participants remained in the study, Figure S1.

\section{Ascertainment of lung cancer}

Participants in screening arm undergo a chest X-ray (one postero-anterior view) at baseline and annually for 2 years; Participants in control arm receive standard medical care. During the follow-up, participants with histologically confirmed lung cancers will be regard as cases. As per the PLCO protocol (NCT01696968), lung cancer diagnosis was confirmed through medical records abstraction and subsequent mortality (from lung cancer or otherwise) was confirmed through a death review committee if available, 
otherwise by death certificate. The ICD codes used in this study is International Classification of Diseases for Oncology, 3rd Edition (ICD-O-3): adenocarcinoma $(\mathrm{AD})$, squamous cell carcinoma (SQ), other non-small cell carcinoma (OTH) and small cell carcinoma (SM). An Annual Study Update was mailed to each participant annually for 13 years to identify all new lung cancers among both screened and control subjects during the trial.

\section{Aspirin use}

The BQ included two questions that pertained to aspirin use. (I) "During the last 12 months, have you regularly used aspirin or aspirin-containing products, such as Bayer, Bufferin, or Anacin?” (Specific instruction was provided to not count aspirin-free products, such as Tylenol or Panadol). (II) "During the last 12 months, how many pills of aspirin or aspirin-containing products did you usually take per day, per week, or per month?" Possible responses were: none, $<2$ pills per month, $2-3$ pills per month, 1 pill per week, 2 pills per week, $3-4$ pills per week, 1 pill per day, or $\geq 2$ pills per day. For this study, we categorized aspirin dose as: (I) no use: no history of aspirin use; (II) irregular use: $<1$ pill per week; (III) low use: $1-4$ pills per week; and (IV) high use: $\geq 1$ pill per day.

\section{Covariate data}

Information regarding the following patient characteristics was obtained from BQ responses: age at time of lung cancer diagnosis (in years), gender, ethnicity, PLCO trial arm, body mass index (BMI), smoking pack-years, family history of cancer (yes or no), and history of diabetes/heart/stroke disease (yes or no). Other intake variables previously reported to have a potential relationship with lung cancer incidence were extracted from DHQ: alcohol (19), total energy (20), vitamin C (21), vitamin E (22), folate (23), calcium (24), red meat (25), processed meat (26), lycopene (27), alpha-carotene (28), fruit (29), vegetables (30), and fat (31).

\section{Statistical analysis}

Age-adjusted incidence rates of lung cancer were determined for categories of aspirin use. Hazard ratio (HR) and 95\% confidence interval $(95 \% \mathrm{CI})$ were defined as the ratio of rate in each category of use compared with rate for no use. We used Cox proportional hazards models to adjust simultaneously for age, smoking characteristics, and other potential confounders. We adjusted the level of age in Ageadjusted analysis (Model 1). In the smoking-adjusted analysis (Model 2), additional covariates were included in Cox models, including pack-years of smoking, gender, race, screening arm, BMI, diabetes, and heart disease and stroke history. Further, dietary factors, which were previously reported to affect lung cancer risk, were also taken into consideration in Dietaryadjusted analysis (Model 3), which additionally adjusted for intake of alcohol, total energy, vitamin $\mathrm{C}$, vitamin $\mathrm{E}$, folate, calcium, red meat, processed meat, lycopene, alpha-carotene, fruit, vegetables, and fat. The association between aspirin frequency and lung cancer risk was further evaluated among participants with different smoked (Never, former, current), and cardiac-cerebral vascular status. The BQ included two questions that pertained to cardiac-cerebral vascular disease. (I) "Did the participant ever have coronary heart disease or a heart attack?" and (II) "Did the participant have a stroke?". Participants with/without cardiac-cerebral vascular disease were analyzed separately, because of the different purpose of aspirin use between two groups (32). Interaction term was incorporated into Cox models to describe synergetic or antagonistic effects among categories of aspirin use, bodyweight, and age. We adopted the propensity score method, which is qualified to adequately adjust for a series of covariates in the Cox proportional hazards regression model (33). Statistical analyses were performed using $\mathrm{R}$ program version 3.4.2. Two-sided $\mathrm{P}$ value of $<0.05$ was considered statistically significant.

\section{Results}

Detailed characteristics of the study population are shown in Table 1, by category of regular aspirin use over the followup period. Results are shown as mean \pm SD for continuous variables and as number (percent) for categorical variables.

\section{High-frequency aspirin use was associated with lung cancer incidence}

Age-adjusted analysis (Model 1) showed a linear trend between aspirin use and lung cancer incidence, with modest positive association ( $\mathrm{HR}=1.07 ; 95 \% \mathrm{CI}, 1.03-1.11$; $\left.\mathrm{P}=2.02 \times 10^{-4}\right)($ Table 2). High-frequency of aspirin use was significantly associated with higher risk of lung cancer $\left(\mathrm{HR}=1.28 ; 95 \% \mathrm{CI}, 1.14-1.44 ; \mathrm{P}=5.11 \times 10^{-5}\right)$. However, effects among irregular and low-frequency aspirin users were not significant when compared with non-users.

In the smoking-adjusted analysis (Model 2), additional 
Table 1 Cohort characteristics, stratified by level of aspirin use

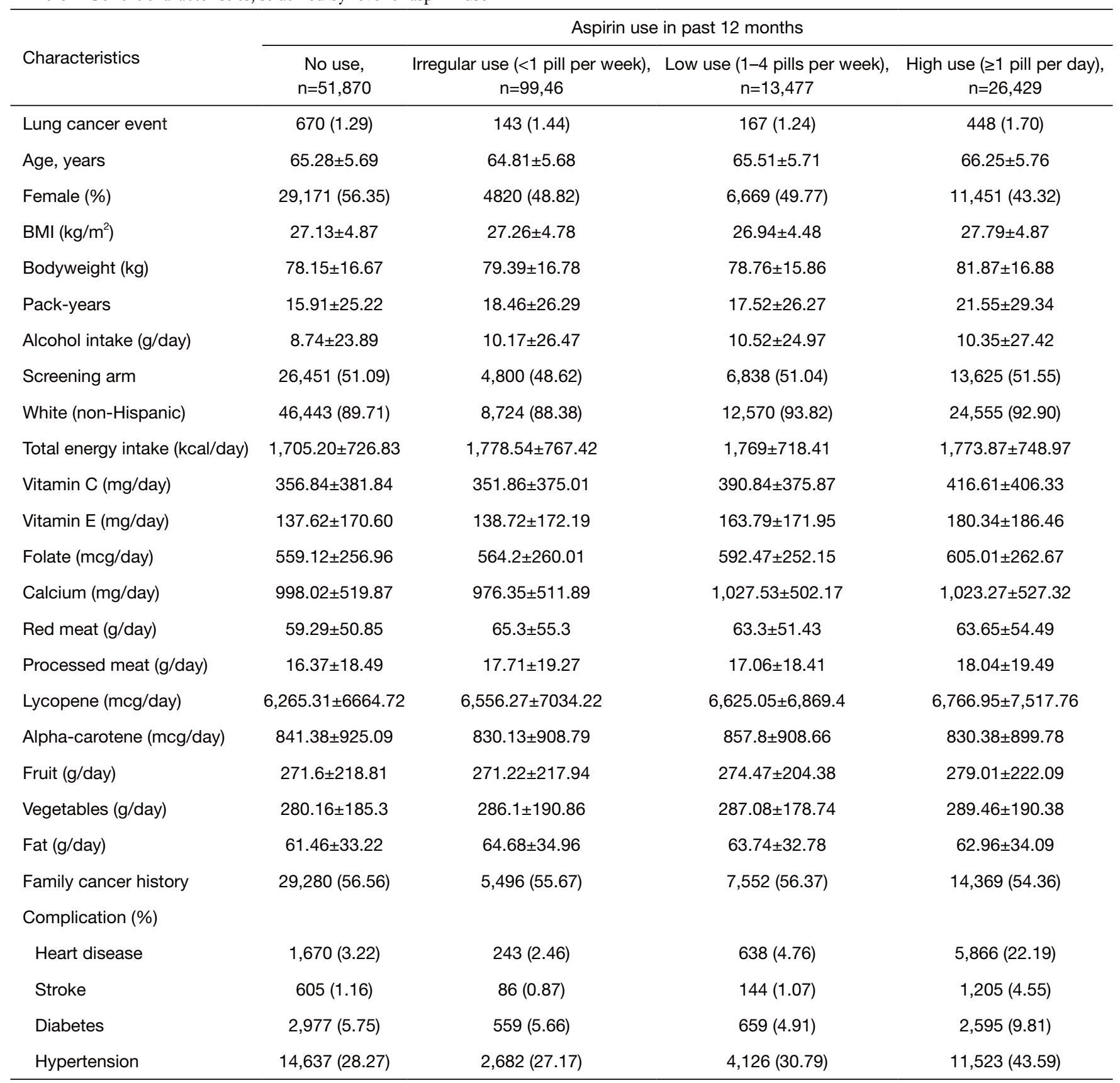

BMI, body mass index.

covariates were included in Cox models, including packyears of smoking, gender, race, screening arm, BMI, diabetes, and heart disease and stroke history. HRs for lung cancer incidence were still not significant for irregularfrequency and low-frequency aspirin use compared to non-users, although high-frequency aspirin use remained significantly associated with the increased risk of lung cancer $\left(\mathrm{HR}=1.29 ; 95 \% \mathrm{CI}, 1.15-1.46 ; \mathrm{P}=3.37 \times 10^{-5}\right)$.

Further, dietary factors, which were previously reported to affect lung cancer risk, were also taken into consideration in Model 3, which additionally adjusted for intake of alcohol, total energy, vitamin C, vitamin E, folate, calcium, 
Table 2 Relationship between aspirin use and lung cancer incidence among all participants

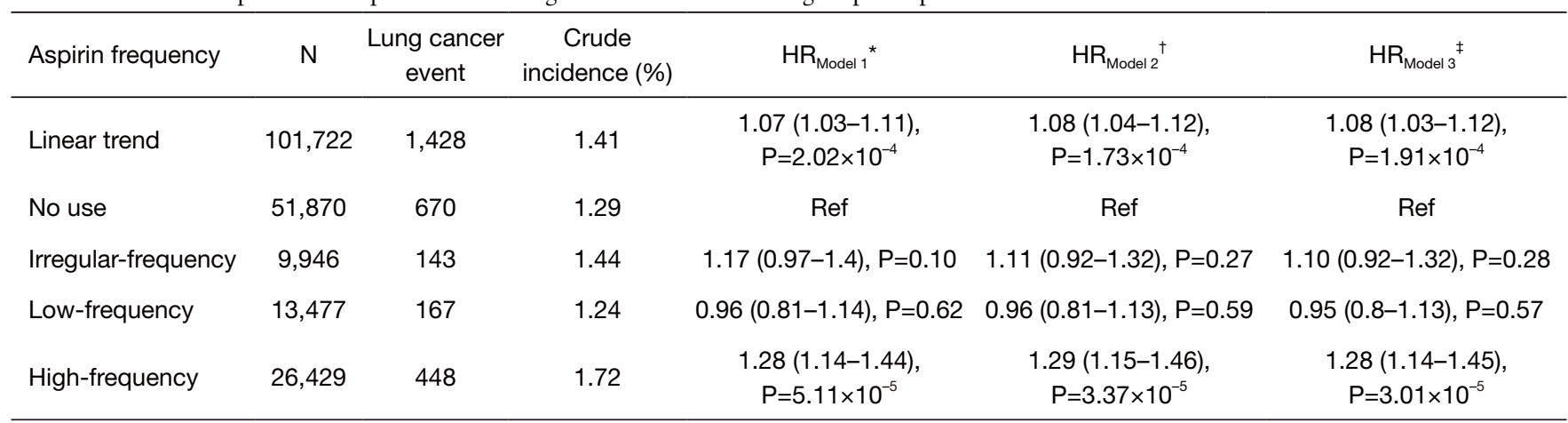

*, Model 1: Cox regression model adjusted for age; ${ }^{\dagger}$, Model 2: Cox regression model adjusted for propensity score calculated using age, pack-years of smoking, gender, race, screening arm, body mass index, diabetes, and heart disease and stroke history; ${ }^{\ddagger}$, Model 3: Cox regression model adjusted for propensity score calculated using age, pack-years of smoking, gender, race, screening arm, body mass index, diabetes, heart disease and stroke history, and intake of alcohol, total energy, vitamin C, vitamin E, folate, calcium, red meat, processed meat, lycopene, alpha-carotene, fruit, vegetables, and fat. Irregular use: 1 pill per week; Low use: 1-4 pills per week; High use: $\geq 1$ pill per day. HR, hazard ratio.

Table 3 Relationship between aspirin use and lung cancer incidence among participants without cardiac-cerebral vascular disease

\begin{tabular}{|c|c|c|c|c|c|c|}
\hline Aspirin frequency & $\mathrm{N}$ & $\begin{array}{c}\text { Lung cancer } \\
\text { event }\end{array}$ & $\begin{array}{c}\text { Crude } \\
\text { incidence (\%) }\end{array}$ & $\mathrm{HR}_{\text {Model } 1}{ }^{*}$ & $\mathrm{HR}_{\text {Model } 2}{ }^{\dagger}$ & $\mathrm{HR}_{\text {Model } 3}{ }^{\ddagger}$ \\
\hline Linear trend & 89,994 & 1,219 & 1.35 & 1.05 (1.01-1.1), $P=0.02$ & 1.05 (1.01-1.1), $P=0.01$ & 1.05 (1.01-1.1), $P=0.01$ \\
\hline No use & 48,971 & 623 & 1.27 & Ref & Ref & Ref \\
\hline Irregular-frequency & 9,346 & 138 & 1.48 & 1.20 (1.00-1.44), $P=0.05$ & 1.13 (0.94-1.36), $P=0.17$ & 1.13 (0.94-1.36), $P=0.18$ \\
\hline
\end{tabular}

${ }^{*}$, Model 1: Cox regression model adjusted for age; ${ }^{\dagger}$, Model 2: Cox regression model adjusted for propensity score calculated using age, pack-years of smoking, gender, race, screening arm, body mass index, diabetes, and heart disease and stroke history; ${ }^{\ddagger}$, Model 3: Cox regression model adjusted for propensity score calculated using age, pack-years of smoking, gender, race, screening arm, body mass index, diabetes, heart disease and stroke history, and intake of alcohol, total energy, vitamin $\mathrm{C}$, vitamin $\mathrm{E}$, folate, calcium, red meat, processed meat, lycopene, alpha-carotene, fruit, vegetables, and fat. Irregular use: 1 pill per week; Low use: 1-4 pills per week; High use: $\geq 1$ pill per day. HR, hazard ratio.

red meat, processed meat, lycopene, alpha-carotene, fruit, vegetables, and fat. Model 3 showed no obvious change in the consequence of aspirin frequency on lung cancer risk. As shown in Table 2, high-frequency aspirin use increased the risk of lung cancer by $28 \%$ ( $\mathrm{HR}=1.28$; $95 \% \mathrm{CI}=1.14-1.45$; $\left.\mathrm{P}=3.01 \times 10^{-5}\right)$. We evaluated the association between highfrequency aspirin use and lung cancer risk among different smoking status. As shown in Table S1, high-frequency aspirin use significantly associated with increased risk of lung cancer for current cigarette smoker ( $\mathrm{HR}=1.30$; $95 \%$ CI, $\left.1.07-1.57 ; \mathrm{P}=6.82 \times 10^{-3}\right)$.

\section{High-frequency aspirin users without cardiac-cerebral vascular disease are susceptible to lung cancer}

Participants with cardiac-cerebral vascular disease had a higher likelihood of high aspirin use (Table 1). Therefore, we stratified the population into two groups according to participants' cardiac-cerebral vascular status and recalculated the association between high-frequency aspirin use and lung cancer risk. For participants without cardiac-cerebral vascular disease, high-frequency aspirin use was significantly associated with the increased risk of lung cancer (Table 3). After adjustment for smoking/ 

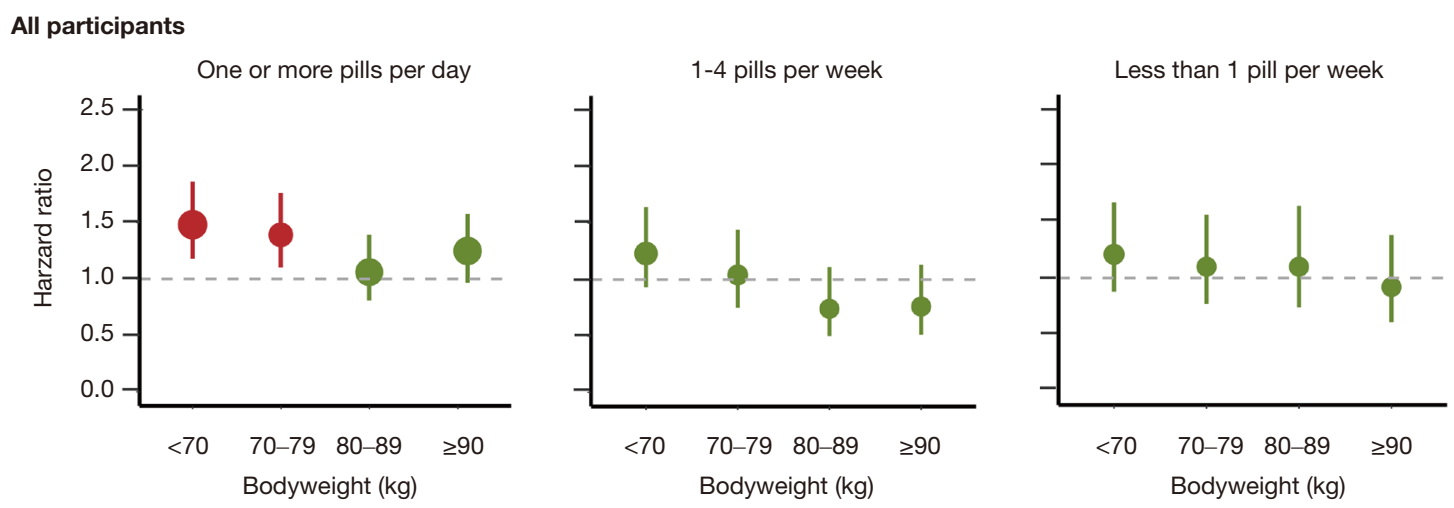

Participants without Cardiac-cerebral vascular disease
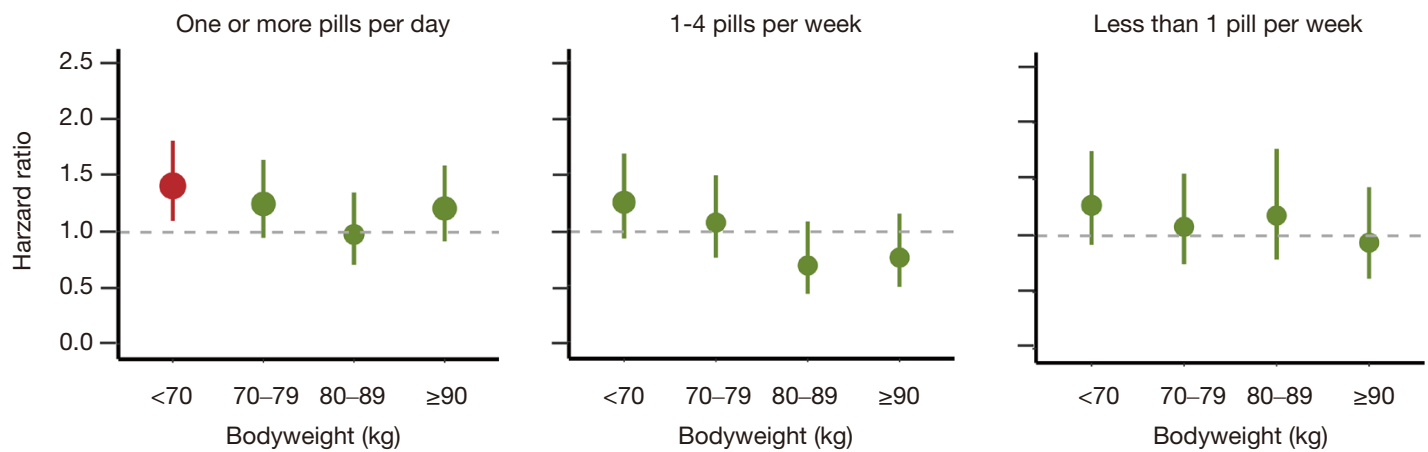

Figure 1 Effect of aspirin use on risk of lung cancer according to bodyweight, compared to no aspirin use, for all participants and for participants without cardiac-cerebral vascular disease. Size of circles representing point estimates of hazard ratios is proportional to inverse of variance of the estimate.

dietary factors, high-frequency aspirin use showed a stably positive association with lung cancer risk ( $\mathrm{HR}=1.22$; $95 \%$ CI, $\left.1.06-1.41 ; \mathrm{P}=4.47 \times 10^{-3}\right)$ compared with aspirin nonusers. Irregular and low-frequency aspirin use did not have a significant association. For participants with cardiaccerebral vascular disease, no significant association with lung cancer risk was observed at any frequency of aspirin use, even if covariates were well-adjusted (Table S2).

\section{Bodyweight modifies the adverse effect of high aspirin use}

To investigate whether the association between aspirin use and lung cancer was modified by bodyweight, we divided participants into bodyweight categories of $<70,70-79$, $80-89$, and $>90 \mathrm{~kg}$. The association between high-frequency aspirin use and lung cancer risk decreased with increasing bodyweight $\left(\mathrm{HR}_{\text {interaction }}=0.96 ; 95 \% \mathrm{CI}, 0.94-0.99\right.$; $\left.\mathrm{P}=1.26 \times 10^{-2}\right)$ in linear model. High-frequency aspirin use and bodyweight of $<80 \mathrm{~kg}$ had a significantly adverse effect for lung cancer risk (Figure 1, Table S3).

For participants without cardiac-cerebral vascular disease, compared with no-users, high-frequency aspirin use among bodyweight of $<70 \mathrm{~kg}$ had a positive association with lung cancer risk (Figure 1 and Table S4), although the linear interaction term (treat aspirin frequency as continuous variable) was not significant $\left(\mathrm{HR}_{\text {interaction }}=0.97 ; 95 \% \mathrm{CI}\right.$, 0.92-1.01; $\mathrm{P}=0.18)$. The association between aspirin use and lung cancer incidence was not significant for participants with cardiac-cerebral vascular disease (Figure S2 and Table S5).

\section{Older age alleviates the adverse association between high- frequency aspirin use and lung cancer incident among individuals with lower bodyweight}

We further evaluated whether high-risk groups of (I) high aspirin users with bodyweight $<80 \mathrm{~kg}$ and (II) high aspirin users without cardiac-cerebral vascular disease and 

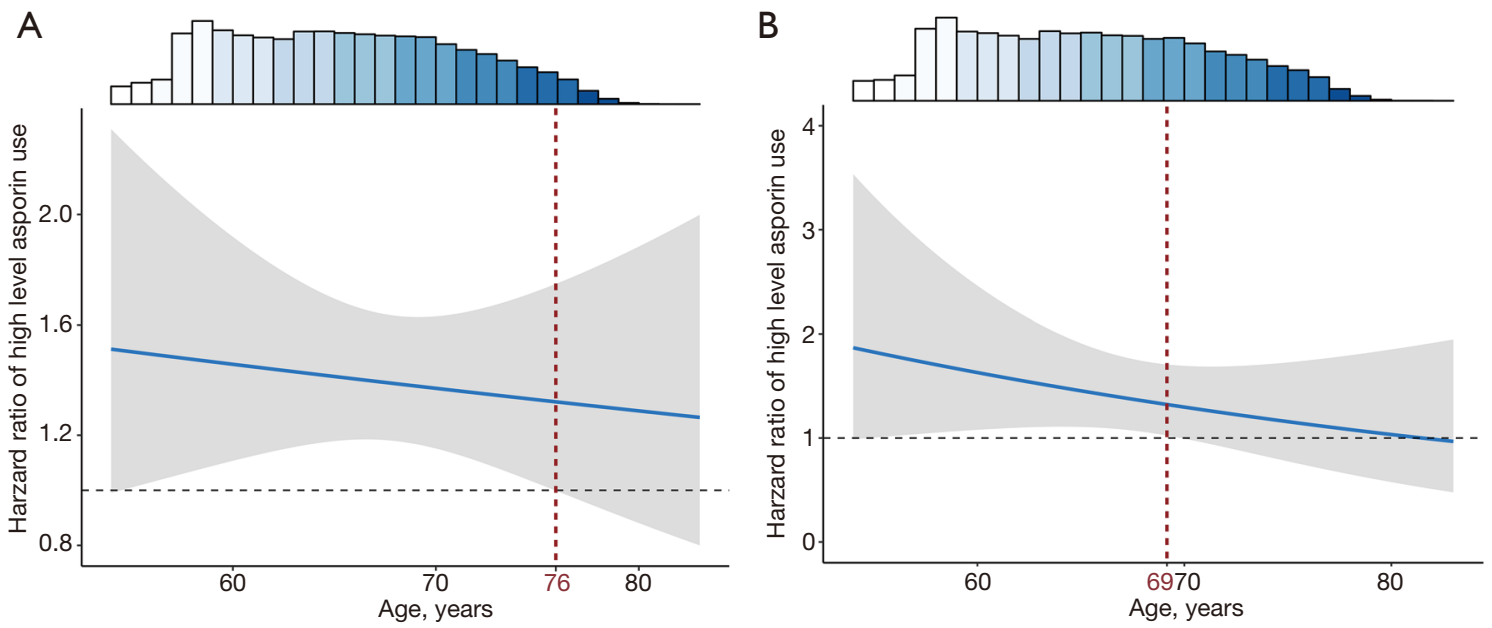

Figure 2 Effect of high-frequency aspirin use ( $\geq 1$ pill per day) compared to no use on risk of lung cancer according to age for (A) all participants and (B) participants without cardiac-cerebral vascular disease. Red dashed lines represent significant cutoff from age; gray dashed lines represent no effect.

bodyweight $<70 \mathrm{~kg}$ had with a "safe age band". Among participants with bodyweight $<80 \mathrm{~kg}$, high aspirin use was associated with decreasing lung cancer risk with increasing age, and the adverse effect of high aspirin use disappeared at $\geq 76$ years of age $\left(\mathrm{HR}_{\text {age }} 76=1.47 ; 95 \%\right.$ CI, $1.25-1.73$; $\mathrm{P}=3.81 \times 10^{-6} ; \mathrm{HR}_{\text {age } 276}=1.13 ; 95 \% \mathrm{CI}, 0.46-2.69 ; \mathrm{P}=0.80$ ) (Figure 2A).

The age modification of adverse association between high-frequency aspirin use and lung cancer incident was also observed among participants without cardiac-cerebral vascular disease. High-frequency aspirin users, without cardiac-cerebral vascular disease and bodyweight $<70 \mathrm{~kg}$, had increased lung cancer risk when age was $<69$ years old $\left(\mathrm{HR}_{\text {age } 69}=1.45 ; 95 \% \mathrm{CI}, 1.05-1.95 ; \mathrm{P}=0.022\right)$ (Figure $\left.2 B\right)$. However, participants with high-frequency aspirin use and age of $\geq 69$ years were not classified as a risk group $\left(\mathrm{HR}_{\text {age }} 69\right.$ $=1.38 ; 95 \%$ CI, 0.99-1.93; $\mathrm{P}=0.054)$, despite relatively low bodyweight $(<70 \mathrm{~kg})$ and no cardiac-cerebral vascular disease.

\section{Discussion}

In this study, a cohort of 101,462 participants from PLCO was used to assess the relationship between aspirin use and lung cancer risk. After comprehensive adjustment for covariates, high-frequency aspirin use was associated with the increased risk of lung cancer, especially for current smoker, and higher bodyweight and older age tended to alleviate this relationship. The results of this study provide new insight for chemoprevention of lung cancer with aspirin.

Aspirin irreversibly and non-selectively inactivates COX, inhibiting both COX-1 and COX-2 enzymes $(3,34)$. However, there is evidence that this inactivating mechanism requires a megadose of aspirin ( $\geq 2$ aspirin tablets per week for 10 years), which indicates that daily regular aspirin use may have no effect on cancer prevention (35-37). On this basis, low aspirin use maybe not be feasible to lower cancer incidence, which has been hinted by the inconsistent results of previous studies.

Bodyweight, instead of BMI, was taken into consideration in this study. Because high-frequency of aspirin should overcome any reduced drug concentration with increasing body size, but might be excessive in patients with low bodyweight because of reduced endothelial prostacyclin production due to high systemic levels of aspirin or possibly because of increased salicylate levels (38). If the effectiveness of lower frequency decreases, and the effectiveness of higher frequency increases, with increasing body size, then weightdose interactions could explain why low-dose aspirin appears to prevent stroke only in women (39), and high doses only in men (40), despite them having similar BMIs. Even so, BMI was also adjusted in Cox analysis. The increased lung cancer risk with high-frequency aspirin use tended to be smaller at higher bodyweight and older age. In other words, there seems to be a dangerous window of aspirin use that is related to bodyweight and age. Consistently, high aspirin use is reported to be an independent factor 
contributing to lung cancer risk in a cohort of U.S. women (15). Our study extends it to a whole-population scale. Our study used propensity score under various confounding and stratification analyses by cardio-cerebrovascular status, which all presented similar evidence. Thus, we have reason to think that our conclusions are reliable.

Despite its strengths, we acknowledge some limitations of our study. (I) Physical activity was not included in this study, which is also important for lung cancer risk. However, measurement of physical activity is more difficult than daily nutrient intake, so quantitative accuracy is difficult to guarantee. (II) Further, we did not focus on lung cancer subtypes. Because nearly $90 \%$ of lung cancer patients in the PLCO trial were diagnosed as non-small cell lung cancer, small sample sizes of other types of lung cancer did not allow robust statistical analysis. (III) Due to the small sample size of the non-cardiac-cerebral vascular disease population, the effect of aspirin use on lung cancer incidence needs further evaluation in future studies. (IV) The underlying biological mechanism, high-frequency aspirin biologically associated with increased the risk of lung cancer, is remain not quite specific. This study just provides a statistical clue for further biological research.

In conclusion, our study provides evidence of interactions among aspirin use, bodyweight, and age for lung cancer risk in U.S. adults. Aspirin should be used carefully for two higher-risk groups of people: (I) individuals with bodyweight $<80 \mathrm{~kg}$ and age $<76$ years; and (II) cardiaccerebral vascular disease patients with bodyweight $<70 \mathrm{~kg}$ and age $<69$ years.

\section{Acknowledgments}

The authors thank the PLCO participants and the hundreds of individuals who worked on the trial throughout its course.

Funding: This study was funded by National Natural Science Foundation of China (81530088 to F.C. and 81973142 to Y.W.), National Key Research and Development Program of China (2016YFE0204900 to F.C.), Natural Science Foundation of Jiangsu Province (BK20191354 to R.Z.), Natural Science Foundation of the Jiangsu Higher Education Institutions of China (18KJB310011 to R.Z.), China Postdoctoral Science Foundation (2018M633767 to R.Z.), US National Institutes of Health (CA209414, CA092824, and ES000002 to D.C.C.), Priority Academic Program Development of Jiangsu Higher Education Institutions (PAPD), and China Scholarship Council
(201906090239 to X.D.). R.Z. was partially supported by the Outstanding Young Teachers Training Program of Nanjing Medical University.

\section{Footnote}

Reporting Checklist: The authors have completed the STROBE reporting checklist. Available at http://dx.doi. org/10.21037/tlcr-20-414

Data Sharing Statement: Available at http://dx.doi. org/10.21037/tlcr-20-414

Conflicts of Interest: All authors have completed the ICMJE uniform disclosure form (available at http://dx.doi. org/10.21037/tlcr-20-414). The authors have no conflicts of interest to declare.

Ethical Statement: The authors are accountable for all aspects of the work in ensuring that questions related to the accuracy or integrity of any part of the work are appropriately investigated and resolved. The study was conducted in accordance with the Declaration of Helsinki (as revised in 2013). All participants provided written informed consent, and the study was approved by both the National Cancer Institute and local Institutional Review Boards (ClinicalTrials.gov registration numbers: NCT01696968).

Open Access Statement: This is an Open Access article distributed in accordance with the Creative Commons Attribution-NonCommercial-NoDerivs 4.0 International License (CC BY-NC-ND 4.0), which permits the noncommercial replication and distribution of the article with the strict proviso that no changes or edits are made and the original work is properly cited (including links to both the formal publication through the relevant DOI and the license). See: https://creativecommons.org/licenses/by-nc-nd/4.0/.

\section{References}

1. Raber I, McCarthy CP, Vaduganathan M, et al. The rise and fall of aspirin in the primary prevention of cardiovascular disease. Lancet 2019;393:2155-67.

2. Steward WP, Brown K. Cancer chemoprevention: a rapidly evolving field. Br J Cancer 2013;109:1-7.

3. Vane JR. Inhibition of prostaglandin synthesis as a mechanism of action for aspirin-like drugs. Nat New Biol 1971;231:232-5. 
4. Cao Y, Nishihara R, Wu K, et al. Population-wide Impact of Long-term Use of Aspirin and the Risk for Cancer. JAMA Oncol 2016;2:762-9.

5. Chubak J, Kamineni A, Buist DSM, et al. Aspirin Use for the Prevention of Colorectal Cancer: An Updated Systematic Evidence Review for the U.S. Preventive Services Task Force. Rockville (MD): Agency for Healthcare Research and Quality (US), 2015.

6. McNeil JJ, Nelson MR, Woods RL, et al. Effect of Aspirin on All-Cause Mortality in the Healthy Elderly. N Engl J Med 2018;379:1519-28.

7. Chubak J, Whitlock EP, Williams SB, et al. Aspirin for the Prevention of Cancer Incidence and Mortality: Systematic Evidence Reviews for the U.S. Preventive Services Task Force. Ann Intern Med 2016;164:814-25.

8. Giardiello FM, Allen JI, Axilbund JE, et al. Guidelines on genetic evaluation and management of Lynch syndrome: a consensus statement by the US Multisociety Task Force on colorectal cancer. Am J Gastroenterol 2014;109:1159-79.

9. Liu Y, Lu Y, Wang J, et al. Association between nonsteroidal anti-inflammatory drug use and brain tumour risk: a meta-analysis. Br J Clin Pharmacol 2014;78:58-68.

10. Bosetti C, Rosato V, Gallus S, et al. Aspirin and cancer risk: a quantitative review to 2011. Ann Oncol 2012;23:1403-15.

11. Cuzick J, Otto F, Baron JA, et al. Aspirin and nonsteroidal anti-inflammatory drugs for cancer prevention: an international consensus statement. Lancet Oncol 2009; 10:501-7.

12. Hernández-Díaz S, Garcia Rodriguez LA. Nonsteroidal anti-inflammatory drugs and risk of lung cancer. Int J Cancer 2007;120:1565-72.

13. Khuder SA, Herial NA, Mutgi AB, et al. Nonsteroidal antiinflammatory drug use and lung cancer: a metaanalysis. Chest 2005;127:748-54.

14. Thorat MA, Cuzick J. Role of aspirin in cancer prevention. Curr Oncol Rep 2013;15:533-40.

15. Feskanich D, Bain C, Chan AT, et al. Aspirin and lung cancer risk in a cohort study of women: dosage, duration and latency. Br J Cancer 2007;97:1295-9.

16. Romano S, Fdez de Gatta MM, Calvo MV, et al. Population pharmacokinetics of amikacin in patients with haematological malignancies. J Antimicrob Chemother 1999;44:235-42.

17. Rothwell PM, Cook NR, Gaziano JM, et al. Effects of aspirin on risks of vascular events and cancer according to bodyweight and dose: analysis of individual patient data from randomised trials. Lancet 2018;392:387-99.

18. Prorok PC, Andriole GL, Bresalier RS, et al. Design of the Prostate, Lung, Colorectal and Ovarian (PLCO) Cancer Screening Trial. Control Clin Trials 2000;21:273S-309S.

19. Freudenheim JL, Ritz J, Smith-Warner SA, et al. Alcohol consumption and risk of lung cancer: a pooled analysis of cohort studies. Am J Clin Nutr 2005;82:657-67.

20. Pillow PC, Hursting SD, Duphorne CM, et al. Casecontrol assessment of diet and lung cancer risk in African Americans and Mexican Americans. Nutr Cancer 1997;29:169-73.

21. Lee DH, Jacobs DR, Jr. Interaction among heme iron, zinc, and supplemental vitamin $\mathrm{C}$ intake on the risk of lung cancer: Iowa Women's Health Study. Nutr Cancer 2005;52:130-7.

22. Chappell J, Eckstein D. MEMORANDUM FOR: science writers and editors on the journal press list : high blood levels of vitamin $\mathrm{E}$ associated with lower incidence of lung cancer. J Natl Cancer Inst 1999;91:1703B.

23. Fanidi A, Muller DC, Yuan JM, et al. Circulating Folate, Vitamin B6, and Methionine in Relation to Lung Cancer Risk in the Lung Cancer Cohort Consortium (LC3). J Natl Cancer Inst 2018;110:57-67.

24. Muka T, Kraja B, Ruiter R, et al. Dietary mineral intake and lung cancer risk: the Rotterdam Study. Eur J Nutr 2017;56:1637-46.

25. Gnagnarella P, Maisonneuve P, Bellomi M, et al. Red meat, Mediterranean diet and lung cancer risk among heavy smokers in the COSMOS screening study. Ann Oncol 2013;24:2606-11.

26. Wang F, Sun GP, Zou YF. Meat consumption and risk of lung cancer: evidence from observational studies. Ann Oncol 2013;24:266-7.

27. Aizawa K, Liu C, Tang S, et al. Tobacco carcinogen induces both lung cancer and non-alcoholic steatohepatitis and hepatocellular carcinomas in ferrets which can be attenuated by lycopene supplementation. Int $\mathrm{J}$ Cancer 2016;139:1171-81.

28. Heber D. Colorful cancer prevention: alpha-carotene, lycopene, and lung cancer. Am J Clin Nutr 2000;72:901-2.

29. Galeone C, Negri E, Pelucchi C, et al. Dietary intake of fruit and vegetable and lung cancer risk: a casecontrol study in Harbin, northeast China. Ann Oncol 2007;18:388-92.

30. Feskanich D, Ziegler RG, Michaud DS, et al. Prospective study of fruit and vegetable consumption and risk of lung cancer among men and women. J Natl Cancer Inst 2000;92:1812-23. 
31. Yang JJ, Yu D, Takata Y, et al. Dietary Fat Intake and Lung Cancer Risk: A Pooled Analysis. J Clin Oncol 2017;35:3055-64.

32. Capodanno D, Mehran R, Valgimigli M, et al. Aspirinfree strategies in cardiovascular disease and cardioembolic stroke prevention. Nat Rev Cardiol 2018;15:480-96.

33. Pirracchio R, Carone M. The Balance Super Learner: A robust adaptation of the Super Learner to improve estimation of the average treatment effect in the treated based on propensity score matching. Stat Methods Med Res 2018;27:2504-18.

34. Machado-Carvalho L, Martín M, Torres R, et al. Low E-prostanoid 2 receptor levels and deficient induction of the IL-1beta/IL-1 type I receptor/COX-2 pathway: Vicious circle in patients with aspirin-exacerbated respiratory disease. J Allergy Clin Immunol 2016;137:99107.e7.

35. Collet JP, Sharpe C, Belzile E, et al. Colorectal cancer

Cite this article as: Dong X, He J, Lin L, Zhu Y, Chen C, Su L, Zhao Y, Zhang R, Wei Y, Chen F, Christiani DC. Association between aspirin use and lung cancer incidence depends on highfrequency use, bodyweight, and age in U.S. adults. Transl Lung Cancer Res 2021;10(1):392-401. doi: 10.21037/tlcr-20-414 prevention by non-steroidal anti-inflammatory drugs: effects of dosage and timing. Br J Cancer 1999;81:62-8.

36. Thun MJ. Beyond willow bark: aspirin in the prevention of chronic disease. Epidemiology 2000;11:371-4.

37. Thun MJ, Namboodiri MM, Calle EE, et al. Aspirin use and risk of fatal cancer. Cancer Res 1993;53:1322-7.

38. Clarke RJ, Mayo G, Price P, et al. Suppression of thromboxane A2 but not of systemic prostacyclin by controlled-release aspirin. N Engl J Med 1991;325:1137-41.

39. Berger JS, Roncaglioni MC, Avanzini F, et al. Aspirin for the primary prevention of cardiovascular events in women and men: a sex-specific meta-analysis of randomized controlled trials. JAMA 2006;295:306-13.

40. Canadian Cooperative Study Group. A randomized trial of aspirin and sulfinpyrazone in threatened stroke. N Engl J Med 1978;299:53-9. 


\section{Supplementary}

Table S1 Relationship between high-frequency aspirin use and lung cancer incidence among participants with different smoked status

\begin{tabular}{lcccc}
\hline Smoked status & $\mathrm{N}$ & Lung cancer event & Crude incidence (\%) & HR \\
\hline Never smoked cigarettes & 48,553 & 115 & 0.24 & $1.15(0.74-1.78), \mathrm{P}=0.54$ \\
Former cigarette smoker & 43,761 & 723 & 1.65 & $1.14(0.96-1.35), \mathrm{P}=0.11$ \\
$<15$ years & 15,116 & 452 & 2.99 & $1.05(0.85-1.29), \mathrm{P}=0.67$ \\
$\geq 15$ years & 27,819 & 261 & 0.94 & $1.18(0.89-1.56), \mathrm{P}=0.26$ \\
Current cigarette smoker & 9,395 & 590 & 6.28 & $1.30(1.07-1.57), \mathrm{P}=6.82 \times 10^{-3}$ \\
\hline
\end{tabular}

Cox regression model adjusted for propensity score calculated using age, pack-years of smoking, gender, race, screening arm, body mass index, diabetes, and intake of alcohol, total energy, vitamin C, vitamin E, folate, calcium, red meat, processed meat, lycopene, alpha-carotene, fruit, vegetables, and fat. High-frequency use: $\geq 1$ pill per day. HR, hazard ratio.

Table S2 Relationship between aspirin use and lung cancer incidence among participants with cardiac-cerebral vascular disease

\begin{tabular}{|c|c|c|c|c|c|c|}
\hline Aspirin frequency & $\mathrm{N}$ & $\begin{array}{l}\text { Lung cancer } \\
\text { event }\end{array}$ & $\begin{array}{c}\text { Crude } \\
\text { incidence } \\
(\%)\end{array}$ & $\mathrm{HR}_{\text {Model 1 }}{ }^{*}$ & $\mathrm{HR}_{\text {Model } 2}^{\dagger}$ & $\mathrm{HR}_{\text {Model } 3}{ }^{\ddagger}$ \\
\hline Linear trend & 9,731 & 197 & 2.02 & 1.06 (0.94-1.19), $P=0.33405$ & 1.05 (0.93-1.18), $P=0.45577$ & $1.04(0.92-1.17), P=0.52911$ \\
\hline No use & 2,080 & 39 & 1.87 & Ref & Ref & Ref \\
\hline Irregular-frequency & 310 & 4 & 1.29 & $0.73(0.26-2.06), P=0.55698$ & 0.73 (0.26-2.05), $P=0.55529$ & $0.71(0.25-1.99), P=0.51526$ \\
\hline
\end{tabular}

*, Model 1: Cox regression model adjusted for age; ${ }^{\dagger}$, Model 2: Cox regression model adjusted for propensity score calculated using age, pack-years of smoking, gender, race, screening arm, body mass index, diabetes; ${ }^{\ddagger}$, Model 3: Cox regression model adjusted for propensity score calculated using age, pack-years of smoking, gender, race, screening arm, body mass index, diabetes, and intake of alcohol, total energy, vitamin C, vitamin E, folate, calcium, red meat, processed meat, lycopene, alpha-carotene, fruit, vegetables, and fat. Irregular-frequency use: 1 pill per week; Low-frequency use: 1-4 pills per week; High-frequency use: $\geq 1$ pill per day. HR, hazard ratio.

Table S3 Association between aspirin use and lung cancer incidence according to bodyweight

\begin{tabular}{|c|c|c|c|c|c|c|c|c|}
\hline Aspirin frequency & Bodyweight $(\mathrm{kg})$ & $\mathrm{N}$ & Lung cancer event & Crude incidence (\%) & $\mathrm{HR}$ & $\mathrm{Cl}$ & $\mathrm{CU}$ & $\mathrm{P}$ \\
\hline \multirow[t]{3}{*}{ High-frequency } & $<70$ & 6,370 & 119 & 1.87 & 1.48 & 1.18 & 1.86 & $6.70 \mathrm{E}-04$ \\
\hline & $70-79$ & 6,450 & 114 & 1.77 & 1.39 & 1.1 & 1.76 & 5.91E-03 \\
\hline & $\geq 90$ & 7,530 & 126 & 1.67 & 1.27 & 1 & 1.61 & $4.98 \mathrm{E}-02$ \\
\hline \multirow[t]{3}{*}{ Low-frequency } & $<70$ & 4,071 & 61 & 1.50 & 1.23 & 0.93 & 1.64 & $1.49 \mathrm{E}-01$ \\
\hline & 80-89 & 2,820 & 29 & 1.03 & 0.74 & 0.5 & 1.11 & $1.45 \mathrm{E}-01$ \\
\hline & $\geq 90$ & 2,940 & 30 & 1.03 & 0.76 & 0.51 & 1.13 & $1.71 \mathrm{E}-01$ \\
\hline \multirow[t]{3}{*}{ Irregular-frequency } & $<70$ & 2,954 & 47 & 1.59 & 1.21 & 0.88 & 1.67 & $2.36 \mathrm{E}-01$ \\
\hline & $70-79$ & 2,417 & 34 & 1.41 & 1.1 & 0.77 & 1.56 & 5.97E-01 \\
\hline & $80-89$ & 2,020 & 30 & 1.49 & 1.1 & 0.74 & 1.64 & $6.27 \mathrm{E}-01$ \\
\hline
\end{tabular}

Cox regression model adjusted for propensity score calculated using age, pack-years of smoking, gender, race, screening arm, body mass index, diabetes, heart disease and stroke history, and intake of alcohol, total energy, vitamin $\mathrm{C}$, vitamin $\mathrm{E}$, folate, calcium, red meat, processed meat, lycopene, alpha-carotene, fruit, vegetables, and fat. Irregular-frequency use: 1 pill per week; Low-frequency use: 1-4 pills per week; High-frequency use: $\geq 1$ pill per day. HR, hazard ratio; $\mathrm{Cl}$, lower confidence interval; CU, upper confidence interval. 
Table S4 Association between aspirin use and lung cancer incidence according to bodyweight among participants without cardiac-cerebral vascular disease

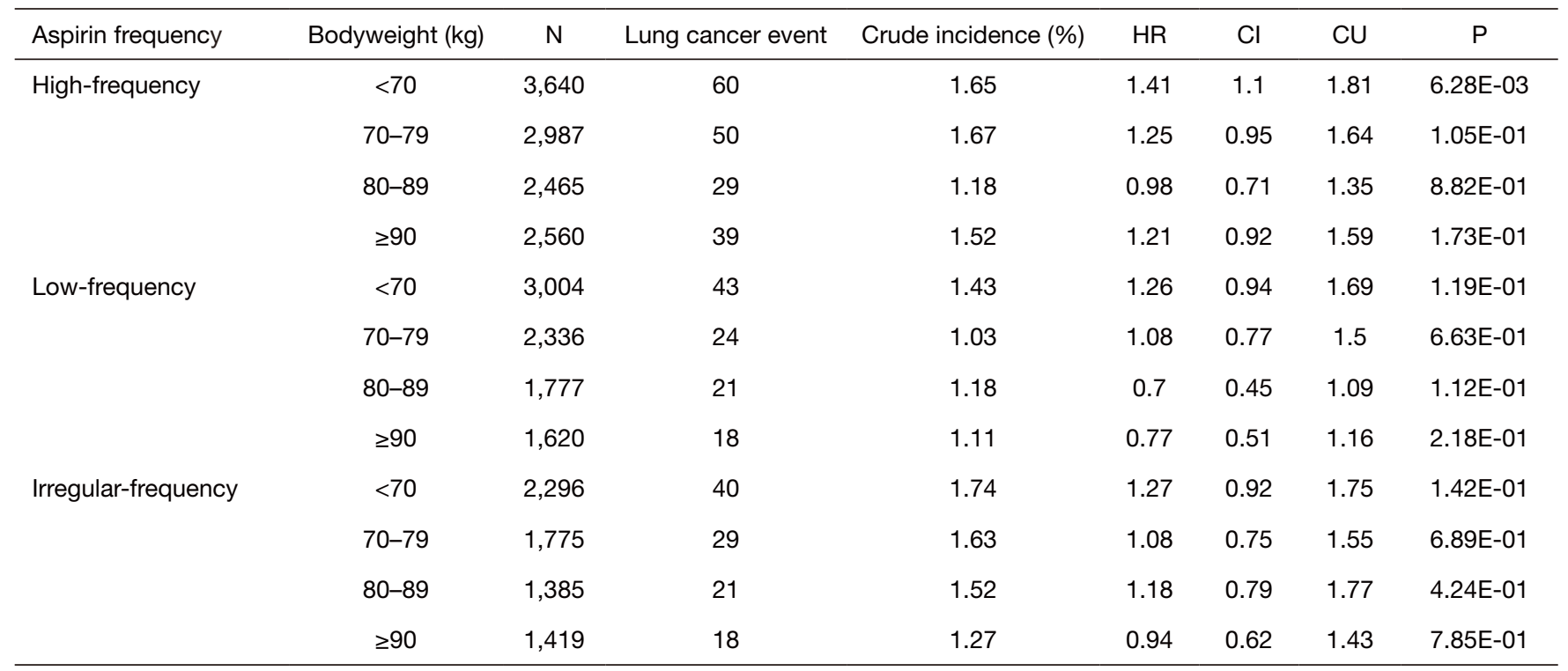

Cox regression model adjusted for propensity score calculated using age, pack-years of smoking, gender, race, screening arm, body mass index, diabetes, and intake of alcohol, total energy, vitamin C, vitamin E, folate, calcium, red meat, processed meat, lycopene, alpha-carotene, fruit, vegetables, and fat. Irregular-frequency use: 1 pill per week; Low-frequency use: 1-4 pills per week; High-frequency use: $\geq 1$ pill per day. $\mathrm{HR}$, hazard ratio; $\mathrm{Cl}$, lower confidence interval; $\mathrm{CU}$, upper confidence interval.

Table S5 Association between aspirin use and lung cancer incidence according to bodyweight among participants with cardiac-cerebral vascular disease

\begin{tabular}{|c|c|c|c|c|c|c|c|c|}
\hline Aspirin frequency & Bodyweight (kg) & $\mathrm{N}$ & Lung cancer event & Crude incidence (\%) & $\mathrm{HR}$ & $\mathrm{Cl}$ & $\mathrm{CU}$ & $P$ \\
\hline \multirow{3}{*}{ High-frequency } & $70-79$ & 3,431 & 63 & 1.84 & 1.31 & 0.65 & 2.65 & 4.50E-01 \\
\hline & $80-89$ & 3,400 & 58 & 1.71 & 0.77 & 0.39 & 1.52 & 4.50E-01 \\
\hline & $\geq 90$ & 4,942 & 87 & 1.76 & 1.22 & 0.62 & 2.43 & 5.66E-01 \\
\hline \multirow{3}{*}{ Low-frequency } & $70-79$ & 1,100 & 20 & 1.82 & 0.55 & 0.12 & 2.53 & 4.43E-01 \\
\hline & $80-89$ & 1,025 & 8 & 0.78 & 1.09 & 0.39 & 3.02 & 8.75E-01 \\
\hline & $\geq 90$ & 1,298 & 12 & 0.92 & 0.6 & 0.13 & 2.77 & 5.17E-01 \\
\hline \multirow[t]{2}{*}{ Irregular-frequency } & $<70$ & 636 & 1 & 0.16 & / & / & / & l \\
\hline & $\geq 90$ & 900 & 1 & 0.11 & / & / & / & / \\
\hline
\end{tabular}

Cox regression model adjusted for propensity score calculated using age, pack-years of smoking, gender, race, screening arm, body mass index, diabetes, and intake of alcohol, total energy, vitamin C, vitamin E, folate, calcium, red meat, processed meat, lycopene, alpha-carotene, fruit, vegetables, and fat. Irregular-frequency use: 1 pill per week; Low-frequency use: 1-4 pills per week; High-frequency use: $\geq 1$ pill per day. $\mathrm{HR}$, hazard ratio; $\mathrm{Cl}$, lower confidence interval; $\mathrm{CU}$, upper confidence interval. 


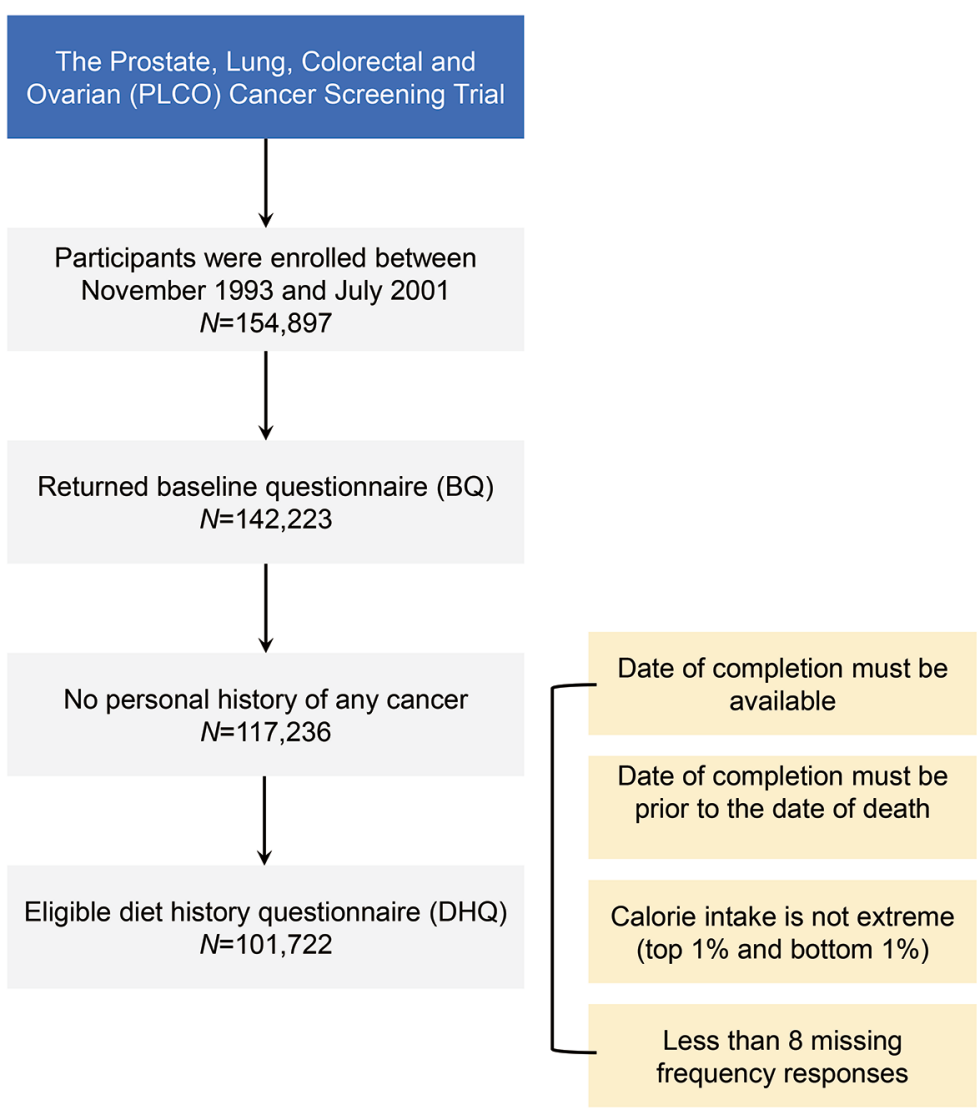

Figure S1 Flowchart for the participants selection.

\section{Participants with cardiac-cerebral vascular disease}
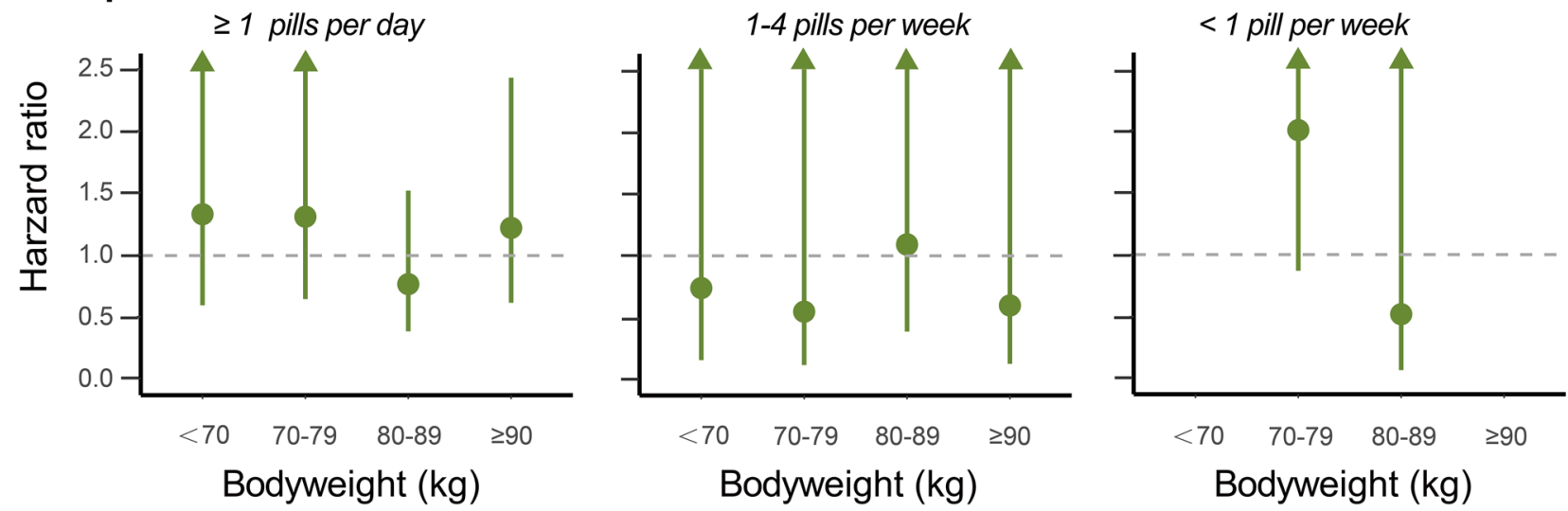

Figure S2 Association between aspirin use and risk of lung cancer according to bodyweight. Size of circles representing point estimates of hazard ratios is proportional to inverse of variance of the estimate. 